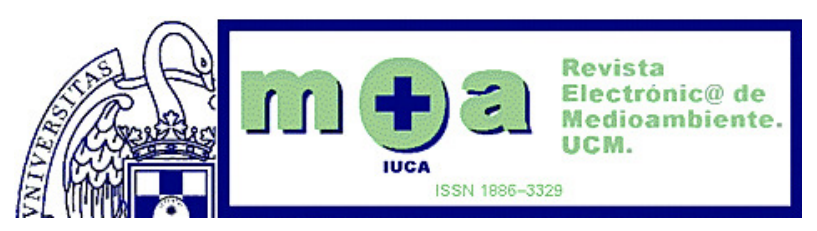

\title{
LAS IMPLICACIONES AMBIENTALES EÓLICAS EN GALICIA
}

\author{
R. M. REGUEIRO FERREIRA \\ Doctora en Economía y profesora de Economía Aplicada \\ rosa.maria.regueiro.ferreira@udc.es
}

Recibido: 20-01-2012

Aceptado:15-06-2012

\section{RESUMEN:}

El desarrollo conseguido por las energías renovables en los últimos años estuvo motivado en parte por el auge conseguido por la energía eólica, que destaca por la no emisión de substancias tóxicas al medio, aunque el proceso de instalación y construcción de los parques eólicos por parte de las empresas promotoras, no es inocuo. A pesar de la existencia de una amplia normativa, a nivel internacional, de España y Galicia, no se cumplen las condiciones mínimas exigidas para garantizar el respeto sobre el ambiente, y una parte importante de los parques eólicos están situados en espacios protegidos de la Red Natura 2000.

Palabras clave: parques eólicos, energía eólica, impacto ambiental, empresas promotoras

\section{The environmental implications of wind energy in Galicia}

\begin{abstract}
:
The development achieved by renewable energy in recent years was driven in part by the growth achieved by the wind, which had the non-emission of toxic substances into the environment, although the process of installation and construction of wind farms by development companies, is not innocuous. Despite the existence of a wide standards, international, Spain and Galicia, are not met minimum requirements to ensure respect for the environment, and an important part of the wind farms are located in protected areas of the Red Natura 2000.
\end{abstract}

Keywords: wind farms, wind energy, environmental, development companies 


\section{Vent dans les conséquences environnementales Galice}

\section{RÉSUMÉ:}

Le développement atteint par les énergies renouvelables ces dernières années a été alimentée en partie par la croissance réalisée par le vent, qui a eu l'émission non de substances toxiques dans l'environnement, bien que le processus d'installation et de construction de parcs éoliens en sociétés de développement, n'est pas anodin. Malgré l'existence d'un éventail de normes, internationales, l'Espagne et la Galice, ne sont pas remplies les exigences minimales pour assurer le respect de l'environnement, et une partie importante des fermes éoliennes sont situées dans des zones protégées de la Croix Rouge Natura 2000.

Mots-clés: fermes éoliennes, l'énergie éolienne, l'environnement des sociétés de développement

\section{LOS PARQUES EÓLICOS: IMPACTOS AMBIENTALES Y POSIBLES MEDIDAS CORRECTORAS}

Un parque eólico se instalará en aquel territorio cuyo régimen de vientos sea de mayor calidad o de calidad adecuada, con respecto a otras alternativas potencialmente viables. Un aerogenerador desarrolla su máxima potencia a una velocidad del viento estimada en $15 \mathrm{~m} / \mathrm{s}$ a $25 \mathrm{~m} / \mathrm{s}$.

Cuando se dispone de una visión clara de los efectos que se van a producir sobre el medio, tanto en la fase de explotación como en la fase de obra, es posible prever las consecuencias sobre los parámetros ambientales que permitirán saber cuáles van a ser los factores más afectados.

Los elementos susceptibles de sufrir impacto son:

a.- medio físico y biológico:

- medio inerte: clima, calidad del aire, hidrología superficial y subterránea, geología, geomorfología.

- medio biótico: flora y fauna

- medio perceptual: unidades de paisaje

b.- elementos socioeconómicos:

- medio socio cultural: gestión territorial y patrimonio cultural

- medio económico: desarrollo económico y población

El establecimiento de medidas correctoras y control, una vez realizada la previsión de alteraciones, debe estar dirigido a minimizar el impacto correspondiente. Tres son las premisas básicas a seguir: 
1.- siempre que fuese posible, es prioritaria la incidencia sobre el diseño del proyecto, de forma que se pueda evitar tal alteración;

2.- si se produce una alteración, puede ser susceptible de tener o no medidas correctoras;

3.- algunas medidas correctoras se articulan bajo el plan de vigilancia, debido a que en el Estudio de Impacto Ambiental no es fácil de prever la magnitud de la alteración.

La efectividad de estas medidas depende de que su ejecución se lleve a cabo durante la obra o en el momento de su finalización.

Los posibles efectos negativos provocados por la implantación de un parque pueden verse mitigados por la utilización de medidas protectoras y correctoras : $_{1}$

- Prácticas generales de obra.

En la fase de construcción se producen, generalmente, las mayores alteraciones sobre el medio físico, debido a la realización de excavaciones, la edificación y la creación de accesos 2 . Se deberían aplicar prácticas organizativas para bloquear los efectos de la calidad del aire, del suelo y el agua. La emisión de polvo y partículas volátiles se podría limitar al centralizar las operaciones de carga y descarga de materiales, y excavaciones . $_{\text {. }}$.

- Gestión de vertidos y residuos.

Cada tipo de residuo generado precisa un tratamiento específico. Se debería comenzar por lograr una minimización del mismo, seguido de un proceso de reciclaje y de realización de vertidos en instalaciones autorizadas.

- Alteración de la vegetación por la apertura de accesos.

La tala de árboles es la práctica más común y extensiva de esta fase del proyecto, por lo que debería llevarse a cabo solo en el área de influencia de los aerogeneradores, de manera que se garantizase a la par un respecto medioambiental y el correcto funcionamiento de las máquinas.

- Plano de accesos.

En numerosas ocasiones no es fácil el acceso a las zonas de obra, por lo que se requieren accesos específicos para que la maquinaria y los materiales lleguen al lugar de utilización. Para eso, deberán de ejecutarse únicamente los movimientos de tierra precisos, sin un tratamiento previo de las mismas, de forma que se reduzcan los riesgos de erosión.

1 Fraga et al.(2007); Gamesa,(2004): el autor expone los principales impactos ambientales, que a su vez son recogidos en la documentación de empresas del sector.

2 Donsión et al.(2002): los autores muestran la importancia de la valoración de los impactos ambientales de esta fase

3 También una práctica utilizada, es el previo riego de las zonas de circulación. Un factor a considerar sería la situación meteorológica, de forma que, en los períodos de inestabilidad se limitaría la ejecución de actividades. 
- Asistencia técnica medioambiental y arqueológica.

La localización de los aerogeneradores va a suponer una modificación definitiva de la zona de implantación del parque, que es responsable a su vez de varios de los impactos antes citados.

- Restaurar las superficies afectadas.

Según el proceso del que se trate, las actividades de restauración a aplicar serían:

- tala de vegetación: plantación de las especies eliminadas;

compactación de terrenos llanos: trabajo superficial, con acercamiento de tierra e hidrosiembra o plantación, si fuese necesario;

- elementos sobrantes de obra y de origen vegetal: retirada y gestión adecuada;

- zonas peladas y riegos erosionados: construcción de muros de retención de piedra, con plantación de plantas y arbustos, así como la instalación de estructuras naturales de estabilización para frenar la erosión;

- eliminación de la cubierta edáfica: restitución de la misma, en orden inverso a la extracción, para poder mantener los niveles de profundidad adecuados, y dejando la capa de tierra más fértil a nivel superficial

- Indemnización por daños provocados.

Los daños diversos provocados por la ejecución de las obras deberán ser reparados en caso de que sea posible, o justamente indemnizados.

- Medidas anticolisión de aves.

La colisión de las aves contra los aerogeneradores no es habitual, si bien se considera que en el diseño de los mismos se deberían evitar superficies planas en las que se posasen las aves, de manera que no se produzcan daños cuando se inicie el funcionamiento de los mismos.

- Reducción del calendario de ejecución de las obras.

El tiempo efectivo de implantación de los elementos de obra en el terreno se tenderá a limitar al estrictamente necesario, de forma que se empiece evitando el retraso en la tramitación de solicitud de concesión de los pertinentes permisos. Sería imprescindible no hacer coincidir el proceso de desarrollo de la obra con los ciclos vitales de las especies de la zona.

- Balizamiento de protección.

Antes del comienzo de la obra, los elementos de interés arqueológico o de otro tipo, deberán ser correctamente señalados, desde un perímetro de protección, de manera que sea fácil su protección y visualización.

- Programa de Vigilancia Ambiental.

El Programa de Vigilancia Ambiental tiene como fin principal controlar el impacto ambiental que se produzca durante las fases de obra y funcionamiento. Se compone de un seguimiento de las obras a realizar y de las 
condiciones ambientales en la fase de explotación por un período de un año desde la fecha de entrega del Acta de Recepción provisional de la obra.

Otro tipo de riesgo afecta solo a las zonas próximas a los aerogeneradores, y que son motivadas, en gran parte, por el desgaste provocado por el uso, como la rotura de palas, la rotura de torre y la caída del personal de mantenimiento.

Para que sea concedida la autorización de instalación del parque eólico, la empresa promotora está obligada a presentar una memoria ambiental, que recoja los efectos potenciales sobre la fauna, la flora, el paisaje y la biodiversidad del área geográfica afectada. Se deduce pues que se concede la licencia de autorización del parque eólico antes de realizar el estudio de impacto ambiental, semejando que la realización de esta memoria es un mero trámite administrativo ${ }_{4}$. En toda la trayectoria eólica de Galicia, no existe caso de denegación de la concesión por razones de afectación ambiental. La explicación de esta situación está en la imposibilidad de afectación sobre esa dimensión estudiada, porque es una superficie limitada, es decir, se fragmenta la superficie total en diferentes parques eólicos, que son estudiados por separado, sin realizarse una agregación de los efectos ambientales sobre el total de la zona afectada . $_{\text {. }}$

Una vez concedida la autorización de instalación, se requiere a la empresa promotora la realización de un estudio de impacto ambiental sobre el ecosistema, la flora y la fauna de la superficie afectada. Así, en lo que respecta al impacto ambiental y, en particular, en el caso concreto de Galicia, se requiere el D.I.A., que se rige por el Decreto 442/1.990, de 13 de septiembre de la Xunta de Galicia, y que constituye el procedimiento administrativo de la Evaluación de Impacto Ambiental en Galicia.

El problema de este procedimiento está radicado en cuatro factores 6 :

- Se realiza la concesión de la autorización antes de evaluar los posibles impactos.

- La fragmentación del territorio en los diferentes parques eólicos impide ver que si se hiciese el cómputo de forma agregada, se constataría la existencia de tal impacto.

- Se pone la atención en la evaluación del comportamiento de las aves, de los murciélagos y de especies de plantas protegidas por la Directiva de Hábitats, pero no se contempla el efecto sobre anfibios y réptiles.

4 García (2005): se muestra la poca valoración que está recibiendo el estudio de impacto ambiental, muy alejado de sus fines reales.

5 Fraga et al.(2007): es la "principal trampa" para esconder el verdadero impacto ambiental, al realizar una segregación de la superficie afectada.

6 Fraga et al.(2007): se explican los principales elementos que restan credibilidad al procedimiento de valoración de impacto ambiental en el proceso de asentamiento de parques eólicos en Galicia. 
- No se realiza el impacto ambiental provocado por las líneas de evacuación del parque.

El Estudio de Impacto Ambiental debe ser una descripción precisa, clara y detallada del proyecto y de sus acciones, especificándose desde la localización del parque, las acciones que se van a llevar a cabo y que producirán tal impacto, así como los materiales y recursos naturales a utilizar y los residuos y vertidos a generar. Paralelamente, se deben postular las alternativas viables y la solución adoptada para poder compatibilizar un buen funcionamiento de las instalaciones, consiguiendo el mínimo impacto ambiental posible. Se añadirá un inventario ambiental, que refleje las condiciones ambientales de la zona antes del comienzo de las obras, y un análisis de las interacciones ecológicas o ambientales. Los impactos habrá que valorarlos, tanto individualmente como globalmente, detallando la solución propuesta para cada alternativa. Un elemento clave es el establecimiento de medidas protectoras y correctoras, encaminadas tanto a minimizar o eliminar los impactos descritos. Finalmente, para garantizar el cumplimiento de dichas medidas será preciso establecer un Programa de Vigilancia Ambiental.

A pesar de la amplia legislación vigente en esta temática, se aprecia una brecha notable en cuanto a calidad y aplicabilidad en muchos de los estudios de impacto ambiental elaborados, impidiendo poder actuar con un límite mínimo de garantías 7 . Conviene destacar como alguna de las deficiencias más notables el desconocimiento de la fauna, de la flora y de los hábitats de las zonas de actuación, la minusvaloración de los efectos negativos sobre la biodiversidad, y la consideración de espacios temporales de estudio muy cortos que no permiten alcanzar un conocimiento real de las poblaciones de fauna y flora de la zona de estudio, entre otros.

\section{PRINCIPAL LEGISLACIÓN GALLEGA APLICABLE EN MATE- RIA DE EVALUACIÓN DE IMPACTO AMBIENTAL EN LA IMPLANTACIÓN DE PARQUES EÓLICOS. LOS PARQUES EÓLICOS EN ZONAS DE LA RED NATURA 2000}

En relación a la legislación aplicable para la regulación de la evaluación del impacto ambiental de los parques eólicos existen diferentes niveles normativos que son de aplicación para aquellos parques asentados en Galicia $_{8}$, siendo las disposiciones europeas y nacionales también de aplicación en otras comunidades autónomas de España.

7 Fraga et al,(2007): se destaca la inseguridad generada por la situación legal que se vive en el sector

8 En el caso de la normativa gallega se incluye alguna norma que no estando en vigor, fue fundamental por la referencia explícita a los parques eólicos. 
En la normativa a nivel europeo, se aprecia una dinámica evolutiva, pasando del reconocimiento de la importancia de la realización de los estudios de impacto ambiental, a poner en marcha mecanismos administrativos para la realización práctica de tales evaluaciones. Se puede destacar la Directiva 97/11/CE de 3 de marzo de 1997, por la que se modifica la anterior Directiva 85/337/CEE relativa a la evaluación de impacto ambiental de los proyectos públicos y privados sobre el medio ambiente y la Directiva 2001/42/CE, de 27 de junio de 2001, por la que se establece el EIA (evaluación de impacto ambiental) obligatoria de los efectos de determinados planes y programas en el medio ambiente ${ }_{10}$, en todas las materias relacionadas con la agricultura, los bosques, la pesca, la energía, la industria, el transporte, la gestión de residuos, la gestión del agua, la planificación urbana y territorial, entre otras actuaciones, y las que impliquen el desarrollo de proyectos incluidos en los Anexos I y II de la Directiva 97/11/CE. Con todo, parece faltar un mecanismo de obligación real por parte de los Estados miembros para que exijan la realización de tales evaluaciones, y el establecimiento de multas y/o penalizaciones para las actividades incumplidoras.

En la normativa a nivel de España, se recoge en el reglamento propio las Directivas Europeas, así como la importancia de realizar un análisis exhaustivo de los impactos sobre el medio ambiente, derivados de las actuaciones industriales. Destacar la Ley 6/2001, de 8 de mayo, por la que se modifica el Real Decreto Legislativo 1302/1986, de 28 de junio, de Evaluación de Impacto Ambiental ${ }_{11}$, que recoge notables avances en materia de infracciones, así como la Ley 9/2006, de 28 de abril, sobre evaluación de los efectos de determinados planes y programas en el medio ambiente ${ }_{12}$. Pero siguen existiendo vacíos legales, en cuanto no se exigen de forma real, y se conceden autorizaciones administrativas de forma previa a la realización del estudio de impacto ambiental.

La principal normativa desarrollada en Galicia que aborda la evaluación del impacto ambiental a provocar en la implantación de parques eólicos se recoge a continuación:

- Decreto 442/1990, de 13 de septiembre de 1990, de Evaluación de Impacto Ambiental: dirigido a los proyectos públicos y privados que realicen obras, instalaciones o cualquier otra actividad de las recogidas en el anexo I, que tengan que ser autorizadas por la Xunta de Galicia. En aquel momento, le correspondía la tramitación administrativa de los expedientes y la elaboración de los pertinentes infor-

9 DOCE L 73, del 14 de marzo del 1997

10 DOCE L197/30, del 21 de julio del 2001

11 BOE no 111, del 9 de mayo del 2001

12 BOE no 102, del 29 de abril del 2006 
mes técnicos, a la Comisión Gallega de Medio Ambiente (dependiente de la Consellería de la Presidencia y Administración Pública).

- Decreto 205/1995, de 6 de julio, por el que se regula el aprovechamiento de la energía eólica ${ }_{13}$ : indicaba que todas aquellas entidades públicas o privadas interesadas en la implantación y explotación de un parque eólico solicitarían su autorización, previa presentación de un "estudio de evaluación de efectos ambientales, que se realizará conforme a lo dispuesto en el Decreto 327/1991, de 4 de octubre, de Evaluación de efectos ambientales para Galicia" y, además, si "por las características del proyecto se viese afectado algún espacio natural incluido en el Registro General de Espacios Naturales de Galicia;...; se estará a lo dispuesto por el Decreto 442/1990, de 13 de septiembre, de Evaluación del impacto ambiental para Galicia, presentando, en su caso, el correspondiente estudio de impacto ambiental". De presentarse varias solicitudes de autorización que afecten a una misma zona, el decreto establecía que se concedería a aquel peticionario que desde la perspectiva técnica, demostrase una mejor relación entre la producción energética y la afectación ambiental, e que también se adaptase mejor a la planificación energética. Este Plan Eólico Estratégico fue aprobado por la Consellería de Industria y Comercio de la Xunta de Galicia en resolución del 29 de diciembre de $1995_{14}$ que, junto con sucesivas normativas, reguló la implantación de parques eólicos en Galicia en los últimos anos. En 1995 se publicó el primer Plan Energético de Galicia (Xunta de Galicia,1995) que contemplaba unas previsiones para el desarrollo de diversas fuentes de energía que, en general fueron superadas.

- Decreto 302/2001, de 25 de octubre, por el que se regula el aprovechamiento de la energía eólica en la comunidad autónoma de Galicia $_{15}$ : en el preámbulo del decreto se afirmaba: "De esta forma, nos encontramos ante una energía moderna, limpia y con una alta aceptación social, que considera a los parques eólicos como instalaciones $y$ empresas limpias, sin consecuencias medioambientales negativas...". Contemplaba la compatibilidad de los aprovechamientos eólicos con los espacios naturales integrados en la Red Natura 2000, al suponer que los valores ambientales de estos no se verían afectados por la construcción de parques eólicos en dichas zonas. Por este motivo, gran parte de los parques que se promovieron desde ese año hasta la entrada del nuevo decreto en 2007 , e incluso alguno autorizado y construido con posterioridad, se localizan en dichos espacios naturales.

- Decreto 242/2007, del 13 de diciembre, por el que se regula el aprovechamiento eólico en Galicia ${ }_{16}$ : en su preámbulo hace una declaración de

13 DOG no. 136, del 17 de julio del 1995

14 DOG. no 51, del 12 de marzo del 1996

15 DOG no 235, del 5 de diciembre del 2001

16 DOG no 2, del 3 de enero del 2008 
intenciones que parecería iniciar un cambio de rumbo en la promoción de esta energía: "el desarrollo eólico deberá ser ejemplar en el plano ambiental: esto significa que la energía eólica, además de resultar limpia por no emitir gases contaminantes a la atmosfera, en su implantación deberá ser extremadamente respetuosa con los valores ambientales hasta el punto de no ocupar espacios de especial protección ambiental". Posteriormente, en las disposiciones referentes a los requisitos ambientales de las instalaciones se exige que todos los proyectos se sometan a evaluación de impacto ambiental, debiendo ser objeto de una declaración de impacto ambiental en la consellería competente en medio ambiente, al tiempo que se excluyen de la implantación de parques eólicos aquellos espacios naturales declarados como zonas de especial protección de los valores naturales para formar parte de la Red Natura 2000 conforme al Decreto 72/2004, de 2 de abril, o normativa vigente en cada momento, excepto los proyectos de repotenciación. La declaración positiva de impacto ambiental es una condición imprescindible para la autorización, por lo que, de resultar negativa, sería causa expresa de denegación. También se incluye en los criterios de valoración la mejor relación entre producción energética y afección ambiental.

- Ley 8/2009, de 22 de diciembre, por la que se regula el aprovechamiento eólico en Galicia y se crean el canon eólico y el Fondo de Compensación Ambiental $_{17}$ : la nueva propuesta de "participación pública" en el proceso de implantación de los parques eólicos consistirá en la formación de un Fondo de Compensación Ambiental, alimentado por la recaudación derivada de la imposición de un canon eólico, descrito como una figura extrafiscal, que penalizará el impacto ambiental ocasionado por dichas instalaciones, en función del número de aerogeneradores. Los titulares de los parques eólicos pagarán cantidades adicionales progresivamente por tramos, con el fin de crear un Fondo de Compensación Ambiental, del que se beneficiarán los municipios afectados por la implantación de parques eólicos en sus dominios, para llevar a cabo actividades respetuosas con el medio ambiente sin concretar. Específicamente, se establece en el artículo 5, que el impacto ambiental será considerado un criterio de planificación del aprovechamiento eólico en Galicia, y en el artículo 29, se indica que entre la documentación a presentar, debe incluirse una previsión del adecuado cumplimiento de las medidas de protección del medio ambiente y de minimización del impacto ambiental. En el artículo 31, establece la exclusión de los territorios en Red Natura para la implantación dos parques eólicos, teniendo en cuenta la normativa vigente en cada momento, y salvo los proyectos de repotenciación. En el artículo 37 , se establece que la D.I.A. se solicitará en función del resultado de la evaluación de impacto ambiental, en función de lo establecido en el Decreto 442/1990, antes citado.

En relación al Decreto 302/2001, y teniendo en cuenta lo recogido también en el Decreto 205/1995, se permitió la localización de parques eólicos en zonas protegidas. Desde 1995, se autorizaron unos 50 parques en 15 
espacios de la Red Natura. Uno de los casos más conocidos y significativos es el de la Serra do Xistral (Lugo) que concentra más de veinte parques en lugares con gran valor ecológico, haciendo dudar de las supuestas ventajas ambientales de esta energía renovable cuando los criterios de su expansión se hacen con una casi nula sensibilidad ambiental al primar criterios productivistas e intereses privados de las empresas energéticas en aras de un declarado interés social y utilidad pública que ignora las demandas sociales, los intereses privados de comunidades de montes y particulares, y hace desaparecer valiosos espacios públicos. La solicitud de informes medioambientales a las empresas en los que indicasen los efectos de los parques supusieron apenas una pequeña traba administrativa, al tener que incluir documentos específicos sobre esta cuestión y ser uno de los criterios de valoración para la autorización (junto con la eficiencia energética de la instalación, el desarrollo y ejecución efectiva de los planes tecnológicos e industriales asociados a los planes eólicos empresariales, la influencia en el desarrollo de la red eléctrica de distribución o transporte, la capacidad técnica y financiera del solicitante, los desarrollos vinculados a terrenos y municipios afectados o el plazo de ejecución del parque), por lo que no supuso consecuencias efectivas en la ocupación de espacios naturales.

A mediados de 2005 hay un cambio en el gobierno gallego (PSOE y BNG, este último tendrá las competencias en energía), iniciándose una revisión de la planificación energética anterior, elaborando un nuevo Plan Energético de Galicia para el período 2007-2012, y promoviendo una nueva normativa eólica.

El Decreto 242/2007 recogía como elemento destacado la existencia de la declaración positiva de impacto ambiental, un avance considerable en la regulación ambiental de la expansión de esta energía, pero resultaba insuficiente al evitar ir más allá de los espacios de Red Natura y considerar otros espacios sometidos a algún otro tipo de protección ambiental que, en algunos casos, constituyen hábitats únicos. Por otra parte se deja a la tramitación de la declaración de impacto las posibles medidas correctoras de impactos ambientales, cuando se podrían exigir requisitos técnicos específicos que, estando sobradamente probados, permiten aplicar un principio de precaución que adelante previsibles problemas sobre el entorno, la flora, la fauna o el paisaje. De todas formas, no puso en marcha toda su potencialidad.

La Ley 8/2009 presenta la figura del canon eólico, que es un reconocimiento explícito de que la forma en cómo se expandió la explotación del recurso eólico no es inocua y que provocó importantes daños que deben ser reparados mediante una compensación monetaria en forma de tributo. De algún modo se opone a la motivación para pagar una prima a las instalaciones eléctricas de régimen especial, promovidas por los efectos ambientales favorables al utilizar fuentes renovables, con la diferencia de que el órgano recaudatorio es en el caso del canon la Xunta de Galicia y quien paga la prima es el Gobierno central. No parece el criterio más acertado relacionar el canon 
solo con el número de aerogeneradores, cuando habría otros criterios razonables como, por ejemplo, su densidad (aerogeneradores $/ \mathrm{km}^{2}$ ), la potencia instalada, la producción u otras consideraciones de índole ambiental. Matizando con mayor detalle la significación económica, fiscal y social del canon, conviene destacar su concepción como elemento de penalización del impacto ambiental provocado. Considerando que en el texto del documento (artículos 15 y 16) se considera la existencia de bonificación por actividades de repotenciación (reducción del número de aerogeneradores, al instalar nuevos aerogeneradores de mayor potencia unitaria), resulta necesario definir en base a qué parámetros se considera la existencia de impacto ambiental. La reducción del número de aerogeneradores puede llegar a disminuir el impacto pero, ¿̇realmente la reducción del número de aerogeneradores y su sustitución por otros de mucha mayor envergadura contribuye a una disminución del impacto visual cuando los nuevos se harán visibles a mayores distancias? La eliminación de aerogeneradores puede disminuir el impacto visual dependiendo de la dimensión de los nuevos aerogeneradores a instalar. A modo ilustrativo, un aerogenerador G-47 de Gamesa, ampliamente extendido en Galicia, tiene un diámetro de palas de 47 metros y tiene una potencia de 750 kW. Un aerogenerador G-850 de Gamesa tiene un diámetro de palas que oscila entre los 52 y los 58 metros. Si se sustituyesen por los nuevos aerogeneradores con potencia de $2,1 \mathrm{MW}$, el diámetro de palas se situaría sobre los 70 metros. Si se sustituyese por aerogeneradores de 4,5 MW operativos en otros países, el diámetro pasaría a ser de 112 metros; si fuese de $5 \mathrm{MW}$, el diámetro sería de 126 metros, y si fuese el aerogenerador experimental de $8 \mathrm{MW}$, el diámetro sería de 160 metros, midiendo cada pala tanto como cada ala de un Airbus380. Debe considerarse que las palas aumentan en longitud y también en amplitud, y que la altura de la torre que soporta la góndola también se incrementaría de forma proporcional. Cabe preguntarse si ante estas actuaciones de repotenciación, realmente se está contribuyendo a una disminución del impacto visual. Tampoco debe olvidarse que cuanto mayor sea el aerogenerador, también mayor debe ser la dimensión de la base de la zapata de hormigón que lo soporta, por lo que la obra de instalación también provocará un impacto mayor sobre el entorno. Por otro lado, se debe considerar que aunque la potencia aumente progresivamente, la producción derivada de ese aumento de potencia se incrementará exponencialmente, por lo que las empresas productoras tienen mayores beneficios empresariales, y no hay una reversión económica directamente proporcional de los mismos sobre la región que alberga el parque eólico. Conviene apuntar, que no se establecen medidas de control sobre el impacto ambiental en todo el proceso de construcción de los parques eólicos. Al no establecer medidas de actuación preventivas ${ }_{18}$,

18 Merino (2007): existen notables controversias sobre la esixencia de la evaluación de impacto ambiental, si bien expertos coinciden en que debería hacerse una valoración en conjunto del impacto ambiental de un Plan Sectorial Eólico, mas que de los parques individualmente 
parece presentarse como una ley que apoya el "todo vale" en términos de lesión ambiental, argumentando que existe la penalización del pago del canon para compensar tal actuación.

La regulación existente a nivel autonómico no marcó impedimentos para que los parques eólicos fuesen instalados en zonas de Red Natura, a pesar de los avances en la materia recogidos en el Decreto 242/2007. Si en septiembre del año 2009 se instalaron en Galicia 3.173,715 MW de origen eóli-

\begin{tabular}{|c|c|c|c|}
\hline ÁREA & NPARQUES EÓLICOS & MW & LIC \\
\hline Serra do Cando & 3 & 79,58 & Serra do Cando \\
\hline Monte Festeiros & 2 & 99 & Serra do Candán \\
\hline Viveiro & 1 & 36,55 & Monte Maior \\
\hline Carba & 1 & 40 & Serra do Xistral \\
\hline Paxareiras II & 1 & 34,8 & Carnota-Monte Pindo \\
\hline Buio I & 1 & 40,3 & Serra do Xistral \\
\hline Buio II & 1 & 20,8 & Serra do Xistral \\
\hline Capelada & 2 & 31,35 & Costa Ártabra \\
\hline Carba & 2 & 44,2 & Serra do Xistral \\
\hline Pena Luisa & 1 & 21,78 & Serra do Xistral \\
\hline Pena Grande & 2 & 30,36 & Serra do Xistral \\
\hline Xistral & 1 & 21,12 & Serra do Xistral \\
\hline Carballeira & 1 & 24,42 & Serra do Xistral \\
\hline Serra de Careón & 2 & 38,7 & Serra do Careón \\
\hline Pena Forcada & 1 & 33,8 & Costa da Morte \\
\hline G3/G16 & 1 & 18,3 & Serra do Xistral \\
\hline LU04-LU05 & 1 & 41,4 & Serra do Xistral \\
\hline LUG4-LUG5 & 1 & 28,8 & Serra do Xistral \\
\hline EEG2-EEG4 & 3 & 58,5 & Serra do Xistral \\
\hline EEG2 & 3 & 66 & Serra do Xistral \\
\hline EEG1 & 2 & 29,95 & Serra do Xistral \\
\hline EEG3 & 4 & 79,5 & Serra do Xistral \\
\hline EEG4 & 1 & 21,75 & Serra do Xistral \\
\hline Somozas & 2 & 38,2 & Xubia-Castro \\
\hline Capelada & 1 & 10,2 & Costa Artabra \\
\hline Montouto & 1 & 20,46 & Serra do Xistral \\
\hline Fiouco & 1 & 24 & Serra do Xistral \\
\hline Serra do Faro & 2 & 62,4 & Monte Faro \\
\hline Serra do Farelo & 1 & 36,8 & Monte Faro \\
\hline Mondoñedo & 1 & 48,43 & Xistral \\
\hline SEN PEE & 3 & 20,475 & Costa da Morte \\
\hline SEN PEE & 1 & 17,56 & Fragas do Eume \\
\hline SEN PEE & 1 & 10,5 & Ortigueira-Mera \\
\hline SEN PEE & 1 & 18 & Brañas do Xestoso \\
\hline TOTAL & 53 & $1.247,285$ & \\
\hline
\end{tabular}

Tabla 1. Afectación de parques eólicos en la Red Natura 2000

Fuente: Simón et al (2010) 
ca, 1.247,285 MW se localizaban en zonas de la Red Natura 2000, representando un $39,3 \%$ de la potencia total instalada ${ }_{19}$. Así se recoge en la tabla 1:

La Serra do Xistral aparece literalmente invadida por el asentamiento de parques eólicos, hecho que se debe al registro óptimo de vientos que se detectó en determinadas zonas de este emplazamiento, tal y como recogió en su momento el Plano de Vientos del Instituto para la Diversificación y el Ahorro de la Energía (IDAE).

Un total de 53 parques eólicos están asentados en zonas pertenecientes a la Red Natura 2000, con un total de 1.247, 285 MW instalados. El ranking por zonas afectadas aparece en la tabla 2:

\begin{tabular}{|l|c|c|}
\hline \multicolumn{1}{|c|}{ LIC AFECTADO } & N $^{\mathbf{0}}$ TOTAL PARQUES EÓLICOS & TOTAL MW \\
\hline Serra do Xistral & 28 & 630,94 \\
\hline Costa da Morte & 4 & 54,275 \\
\hline Serra do Cando & 3 & 79,58 \\
\hline Monte Faro & 3 & 99,2 \\
\hline Costa Artabra & 3 & 41,55 \\
\hline Serra do Candán & 2 & 99 \\
\hline Serra do Careón & 2 & 38,7 \\
\hline Xubia-Castro & 2 & 38,2 \\
\hline Xistral & 1 & 48,43 \\
\hline Monte Maior & 1 & 36,55 \\
\hline Carnota-Monte Pindo & 1 & 34,8 \\
\hline Brañas de Xestoso & 1 & 18 \\
\hline Fragas do Eume & 1 & 17,56 \\
\hline Ortigueira-Mera & 1 & 10,5 \\
\hline TOTAL & 53 & $1.247,285$ \\
\hline
\end{tabular}

Tabla 2. Ranking de espacios de Red Natura 2000 por No parques eólicos y potencia instalada

Fuente: Elaboración propia a partir de Simón et al (2010)

La afectación sobre la Serra do Xistral queda reflejada de una forma clara, tanto en número de parques eólicos asentados (28) como en potencia, con un 54,7\% de la potencia instalada en Red Natura $2000_{20}$. Otras zonas, como la Costa da Morte, la Serra do Cando y Monte Faro, presentan un número de parques eólicos más reducido, aunque la significación en términos de potencia entre ellos no sigue el mismo nivel decreciente. El hecho de que en una zona existan menos parques eólicos asentados con mayor potencia, puede deberse a que los aerogeneradores instalados

19 Simón et al.(2010): los autores cifran en términos de potencia el asentamiento de parques eólicos en zonas protegidas de la Red Natura 2000.

20 Simón et al.(2010): en la documentación original, se presentaban los parques eólicos de forma individualizada, por lo que se realizó una agregación de los mismos para ver su significación real por zonas geográficas de la Red Natura 2000 
registran una mayor potencia nominal, por lo que son más grandes o que el parque eólico disponga de un mayor número de aerogeneradores. En cualquier caso, el impacto ambiental negativo está fuera de toda duda. Por otra parte, en la Ley de Aprobación de los Presupuestos Generales de Galicia para el año 2012 se incluirá una modificación que ya ha sido seriamente criticada, entre otras instituciones, por el Colegio Oficial de Biólogos de Galicia: la no obligatoriedad de realizar los estudios de impacto ambiental en los parques eólicos de dimensión supramunicipal.

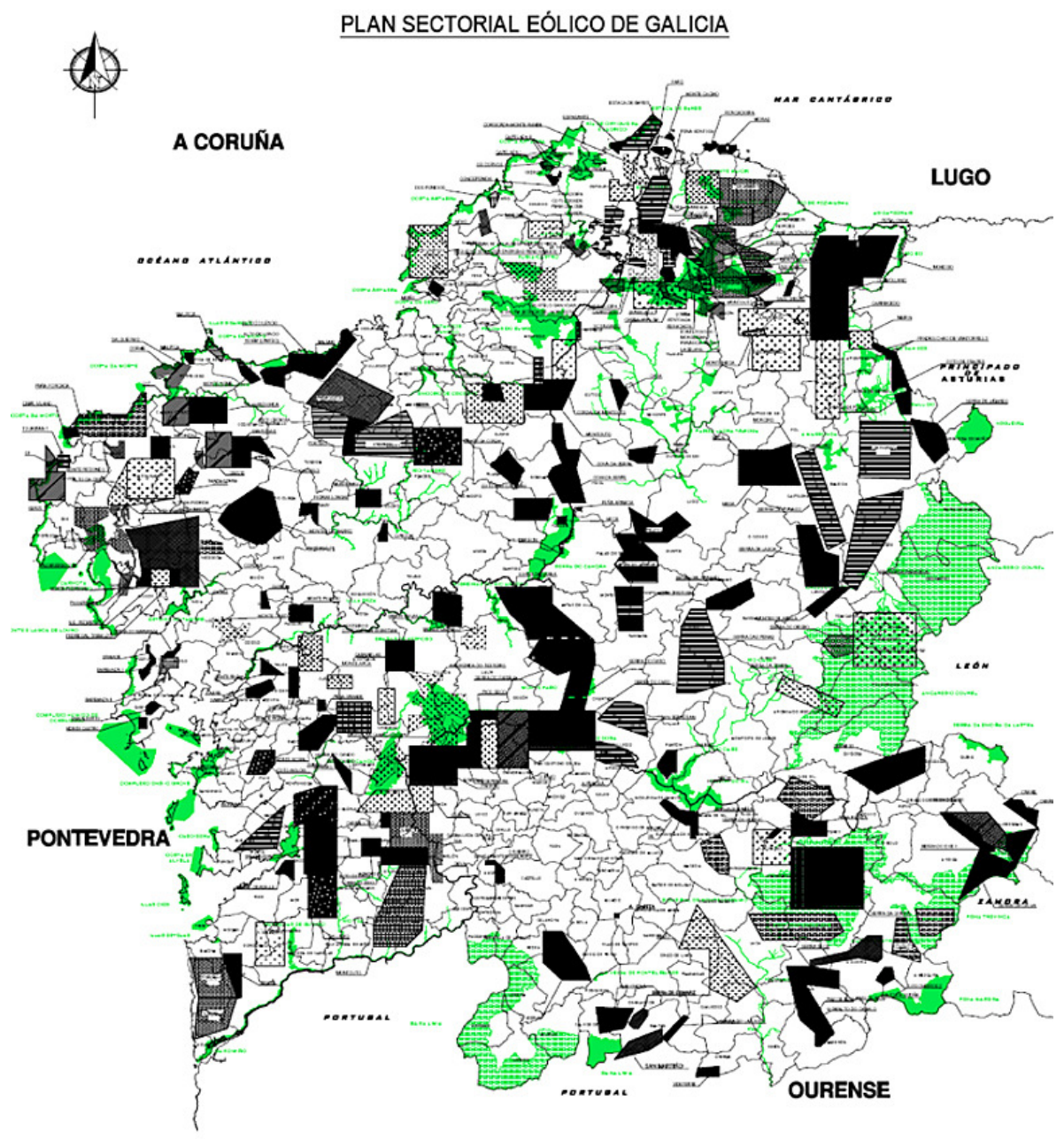

Mapa 1. Plan sectorial eólico con Red Natura.

Fuente: Inega (2010) 


\section{CONCLUSIONES}

El proceso de implantación de parques eólicos por parte de las empresas promotoras no es inocuo con el ambiente, y a pesar de la existencia de una amplia y variada legislación sobre esta temática, las lesiones ambientales se están produciendo constantemente. En relación a Galicia, resulta evidente que tanto en los decretos como en la Ley existen diferentes líneas de actuación, y de modo notable, en relación a la afectación ambiental por parte de las normativas analizadas.

A pesar de más de una década de desarrollo normativo en Galicia, y siendo una comunidad autónoma pionera en el aprovechamiento eléctrico de la energía eólica, sigue existiendo un gran vacío legal en materia ambiental en el que se amparan las empresas energéticas para llevar adelante sus proyectos. La administración competente en energía tendió a una interpretación extremadamente productivista, asumiendo sin problemas que el incremento de la potencia y la producción eólica eran netamente positivas en términos ambientales, al margen del modo en cómo se posibilitase dicho incremento.

La respuesta normativa parece seguir agarrada a una perspectiva productivista, ahora ligeramente teñida de un color más ambiental en el que, como mucho, se deja cierto papel determinante a la administración competente en la materia. Este modo de planificar el desarrollo de las energías renovables en el que no se acaba de incorporar como uno de sus ejes centrales la variable ambiental, está perjudicando la percepción social sobre el uso de energías renovables que deriva de la contradicción manifiesta entre un discurso pseudoecologista en las motivaciones que se expresan en la normativa y las disposiciones que regulan su uso. Esta situación se agrava cuando la sociedad no tiene canales para la participación en el desarrollo de las renovables, quedando relegados a un papel pasivo, de afectados, frente a un papel activo, de agresores y beneficiarios, de las empresas y la administración. Son algunos de los efectos de la inexistencia de una política sectorial global en relación a los asentamientos eólicos.

\section{BIBLIOGRAFÍA}

-Consellería de Industria y Comercio (1995): Decreto 205/1995, de 6 de julio, por el que se regula el aprovechamiento de la energía eólica en la Comunidad Autónoma de Galicia. Diario Oficial de Galicia, 17 de julio del 1995 (núm. 136). -Consellería de Industria y Comercio (1995): Plan Eólico Estratégico.Diario Oficial de Galicia, 12 de marzo de 1996, no 51.

-Consellería de Industria y Comercio (2001): Decreto 302/2001, de 25 de octubre, por el que se regula el aprovechamiento de la energía eólica en la Comunidad Autónoma de Galicia. Diario Oficial de Galicia, 5 de diciembre, no. 235. 
-Consellería de Innovación e Industria (2007): Decreto 242/2007, de 13 de diciembre, por el que se regula el aprovechamiento de la energía eólica en Galicia.Diario Oficial de Galicia, 3 de enero del 2008, no. 2.

-Consellería de Medio Ambiente (1990): Decreto 442/90, del 13 de septiembre del 1990, de evaluación de impacto ambiental.Diario Oficial de Galicia, 25 de septiembre, no. 188.

-Consejo Europeo (1992): Directiva 92/43/CEE del Consejo, del 21 de mayo del 1992, relativa a la conservación de los hábitats naturales y de la fauna y flora silvestres. Diario Oficial de las Comunidades Europeas DOL 206, del 22 de junio de 1992, y con dictámenes rectificativos en el DOL 59 del 8 de marzo del 1996 e en el DOL 31 del 6 de febrero del 1998.

-Consejo Europeo (1997): Directiva 97/11/CE de 3 de marzo do 1997, por la que se modifica la anterior directiva $85 / 337 /$ CEE, relativa a la evaluación del impacto ambiental de los proyectos públicos y privados sobre o medio ambiente. Diario de las Comunidades Europeas, L73, del 14 de marzo de 1997.

-Donsión M.P. et al, (2002): "La energía eólica. Impactos ambientales". Revista Energía, febrero, n²1, p. 119-124.

-Fraga López, Francisco; Martínez Iglesias, José Manuel, e Proupín Castiñeiras, Jorge(2007): "Energías renovables: ¿la energía del futuro?".Curso de verán 2007. Servizo de Publicacións USC.

-Gamesa Energía (2004): www.gamesa.es

-García Arresse A. (2005): Evaluación de impacto ambiental de parques eólicos en Galicia. Universidad de Santiago de Compostela.

-Jefatura del Estado (2001): Ley 6/2001, del 8 de mayo, por la que se modifica el Real Decreto Legislativo 1302/86 del 28 de junio, de Evaluación de Impacto Ambiental. BOE. Boletín Oficial del Estado, del 9 de mayo, $\mathrm{n}^{\circ} .111$.

-INEGA (2010): Mapas Plano Sectorial Eólico. Disponible en la rede: http://www.inega.es/inega/2007/upload/des/244-d-planos.pdf -Merino, Luis (2007):"Eólica y conservación de la naturaleza. Los más críticos lo ven así". Revista Energías Renovables no 56, p. 54-56.

-Ministerio de Medio Ambiente (2006): Ley 9/2006, del 28 de abril, sobre Evaluación de los efectos de determinados planes y programas en el medio ambiente. Boletín Oficial del Estado, 24 de abril, no 102.

-Ministerio de Obras Públicas y Urbanismo (1988): Real Decreto 1131/1988, del 30 de septiembre, por el que se aprueba el Reglamento para la ejecución do Real Decreto Legislativo 1302/1986, del 28 de junio, de la evaluación del impacto ambiental. Boletín Oficial del Estado, 5 de octubre, no.239.

-Parlamento Europeo y Consejo Europeo (2001): Directiva 2001/42/CE, del 27 de junio del 2001, por la que se establece la EIA obligatoria de los 
efectos de determinados planes y programas en el medio ambiente. Diario Oficial de las Comunidades Europeas, L 197/30, del 21 de julio.

- Simón Fernández, Xavier et al, (2010): Os plans eólicos empresariais no sector eólico galego. Consello Social-Universidad de Vigo.

- Xunta de Galicia (2009): Ley 8/2009, del 22 de diciembre, por la que se regula el aprovechamiento eólico en Galicia y se crean el canon eólico y el Fondo de Compensación Ambiental.Diario Oficial de Galicia.29 de diciembre, no 252. 